\title{
Guest Editorial: Advances in the management of acquired aplastic anemia
}

\author{
Shinji Nakao
}

Received: 1 April 2013/Revised: 3 April 2013/Accepted: 3 April 2013/Published online: 21 April 2013

(C) The Japanese Society of Hematology 2013

Acquired aplastic anemia (AA) is a poorly characterized disease. The pathophysiology of AA can be simply explained by a decrease in the number of hematopoietic stem cells (HSCs); however, how the HSCs are lost remains unknown, despite vigorous efforts to clarify the mechanism. It has been considered that an autoimmune mechanism, such as a cytotoxic T cell attack against HSCs, causes AA. The most compelling evidence for such an immune mechanism, however, is derived from clinical observations that approximately two-thirds of AA patients improve with immunosuppressive therapy (IST). The management of AA improved greatly over the past 20 years, mainly owing to the introduction of IST, including anti-thymocyte globulin (ATG) and cyclosporine, and advances in allogeneic bone marrow transplantation (BMT) from unrelated donors.

However, a number of issues still need to be resolved regarding the clinical management of AA. First, the $60-70 \%$ response rate of patients with AA to IST is far from satisfactory. Some patients may benefit from a second round of IST, but this can occur at the cost of complications associated with immunodeficiency. Thus, an optimal treatment regimen for IST needs to be developed to improve the response rate. The lower efficacy of rabbit ATG (Thymoglobulin) compared to horse ATG (ATGAM) was recently reported by the NIH and EBMT groups, and raised concerns about the efficacy of

S. Nakao $(\square)$

Cellular Transplantation Biology, Kanazawa University

Graduate School of Medical Science, 13-1 Takaramachi,

Kanazawa, Ishikawa 920-8641, Japan

e-mail: snakao8205@staff.kanazawa-u.ac.jp rabbit ATG in Asian countries, where horse ATG is unavailable. However, the response rates of Korean and Chinese AA patients to rabbit ATG were reported to be similar to those for horse ATG.

The limited success rate of IST in patients with AA may be due to an underlying non-immune mechanism, rather than the inadequacy of immunosuppression. Thus, it is necessary to distinguish between immune and non-immune mechanisms in patients with AA. The method used to detect the immune pathophysiology of BM failure is even more important for distinguishing between AA and lowrisk myelodysplastic syndrome (MDS). In addition to markers previously reported to be useful for identifying immune pathophysiology, a recent study reported a promising role of determining the plasma thrombopoietin levels for this purpose.

Another important issue in the management of AA is how to identify patients with forms of the disease with a high risk of evolving into MDS. Several molecular markers, such as telomere attrition and the presence of genetic alterations associated with the pathogenesis of MDS, have been proposed as potentially useful for predicting transition to MDS. Detection of preleukemic features in patients with AA will allow physicians to choose BMT earlier in the course of the disease. Finally, the optimal conditioning regimens for allogeneic BMT have not yet been established, particularly for BMT from unrelated donors. Although the inclusion of ATG in the conditioning regimen improved the outcome of BMT for AA patients, it increases the risk of Epstein-Barr virus (EBV)-related lymphoproliferative disorders (LPD), and may not be sufficiently potent to abolish graft-versushost disease (GVHD), which does not help patients with AA. Other methods of in vivo T-cell depression, such as the administration of alemtuzumab, may improve the 
survival of patients undergoing BMT by lowering the incidence of EBV-related LPD as well as of GVHD.

The Progress in Hematology articles in this issue highlight problems associated with AA. These reviews will hopefully improve the management of AA by readers, and help to unravel the mysteries of this disease. 